\title{
Amrubicin Monotherapy for Elderly Patients with Previously Treated Lung Cancer
}

\author{
Makoto Nakao, Tetsuya Oguri, Takeshi Suzuki, Eiji Kunii, Yuki Tomita, Yasuhito Iwashima, \\ Mikinori Miyazaki, Ken Maeno, Shigeki Sato and Ryuzo Ueda
}

\begin{abstract}
Objective The novel anthracycline agent amrubicin, has been approved in Japan to treat small and nonsmall cell lung cancers (SCLC and NSCLC). The present study evaluates the toxicity and effect of amrubicin especially in elderly patients with previously treated lung cancer.

Patients and Methods This retrospective study analyzed data from 51 patients $(<70$ years of age, $n=29$; $\geq 70$ years of age, $n=22$ ) with lung cancer (NSCLC, $n=21$; SCLC, $n=30$ ) who were treated with amrubicin at our hospital, between July 2003 and October 2009. All patients had recurrent or refractory lung cancer after one or more chemotherapy regimens. We compared the outcomes of patients younger and older than 70 years of age. Amrubicin (30-40 mg/m $/ \mathrm{day})$ was infused depending on patient performance status and laboratory data over a period of 5 minutes on days 1-3, with courses repeated at intervals of at least 3 weeks. The dose was modified according to myelosuppression.

Results The mean number of treatment cycles, mean dose and mean interval of amrubicin administration did not significantly differ between patients aged $<70$ and $\geq 70$ years. The rate of hematological toxicities ( $\geq$ Grade 3 ) also did not significantly differ between the two age groups (leukopenia, $48.3 \%$ and $59.1 \%$ for age $<70$ and $\geq 70$ years, $\mathrm{p}=0.573$; neutropenia, $65.5 \%$ vs. $77.3 \%, \mathrm{p}=0.536$; anemia, $20.7 \%$ vs. $22.7 \%$, $\mathrm{p}=$ 1.000 ; thrombocytopenia, $13.8 \%$ vs. $31.8 \%, \mathrm{p}=0.173$ ). The incidence of grade $2-4$ non-hematological toxicities also did not significantly differ between the groups. The response rate of SCLC and disease control rate of NSCLC were similar in the younger and older groups.

Conclusion Amrubicin monotherapy might be equally tolerated by elderly and younger patients. Further studies are needed to investigate the benefit of amrubicin monotherapy among elderly patients with previously treated lung cancer.
\end{abstract}

Key words: chemotherapy, elderly, lung cancer, amrubicin

(Inter Med 49: 1857-1862, 2010)

(DOI: 10.2169/internalmedicine.49.3606)

\section{Introduction}

Amrubicin is a synthetic anthracycline anticancer drug and a potent topoisomerase II inhibitor (1). Its antitumor effects are thought to be derived from a metabolic reduction in tumor cells and conversion to the active metabolic amrubicinol, which more potently inhibits cell growth by about 200-fold compared to the parent compound (1). Amrubicin has more potent antitumor effects in vivo, with less cardio-, hepato- and nephrotoxicity than other anthracycline antican- cer drugs, such as doxorubicin (1).

Amrubicin has been approved for treating small cell lung cancer (SCLC) and non-small cell lung cancer (NSCLC) in Japan and it is generally administered to patients who have previously been treated for lung cancer. Amrubicin can be administered within 5 minutes, which is convenient for outpatients. The response rates of previously treated NSCLC and SCLC are 10.2-13.5\% and 38-53.1\%, respectively (1-5). The most frequent drug-related adverse event is myelosuppression, and the incidence of non-hematological toxicities is low (1-7). Although an acceptable toxicity of amrubicin 
monotherapy in previously treated lung cancer patients has been determined, the dose of amrubicin in such patients must be reduced $(3,5-7)$.

Meanwhile, the risk of lung cancer clearly increases with advancing age and about $40 \%$ of patients are aged 70 years or older (8). Moreover, elderly patients tend to have poor tolerance to chemotherapy, and thus need to be managed based on individual parameters such as performance status, extent of metastatic disease, quality of life and laboratory data (9-11). Whether or not standard chemotherapy in elderly patients is always safe for use in clinical practice is unclear (12).

The toxicity and effect of amrubicin monotherapy in elderly patients who have been previously treated for lung cancer has not been fully evaluated. We therefore retrospectively assessed the safety and value of amrubicin among elderly patients who had previously been treated for lung cancer.

\section{Patients and Methods}

\section{Patient selection}

We retrospectively reviewed all patients who had been previously treated for lung cancer and who received amrubicin monotherapy (30-40 mg/m $/$ day) after one or more chemotherapy regimens between July 2003 and October 2009 at Nagoya City University Hospital. All of the patients who received amrubicin monotherapy met the following criteria: histologically and/or cytologically proven NSCLC or SCLC; performance status (PS) 0-3 on the Eastern Cooperative Oncology Group scale; adequate bone marrow reserve (white blood cell count $\geq 3,000 / \mathrm{mm}^{3}$, absolute neutrophil count $\geq$ $1,500 / \mathrm{mm}^{3}$, platelet count $\geq 100,000 / \mathrm{mm}^{3}$ ); normal liver function (total serum bilirubin $\leq 1.5 \mathrm{mg} / \mathrm{dL}$, and aspartate transaminase, alanine transaminase less than twice the upper limit of the normal range); normal renal function (serum creatinine $\leq 1.5 \mathrm{mg} / \mathrm{dL}$ ); normal cardiac function (left ventricular ejection fraction $\geq 50 \%$ ) and no pulmonary fibrosis/ interstitial pneumonia. The patients were then assigned to younger (age $<70$ years) or elderly (age $\geq 70$ years) groups.

\section{Treatment methods}

Amrubicin $\left(30-40 \mathrm{mg} / \mathrm{m}^{2}\right)$ was administered intravenously as a 5-min infusion in normal saline on days 1-3. All courses were repeated at intervals of at least 3 weeks or longer. Each treatment was repeated until disease progression became obvious, the patient refused to proceed, or toxicity became intolerable. Patients were required to have a white blood cell count of $\geq 3,000 / \mathrm{mm}^{3}$, and a platelet count of $\geq 100,000 / \mathrm{mm}^{3}$ without any non-hematologic toxicities of grade 2 or worse to start the next course. The patients were premedicated with corticosteroid and administered with an antiemetic 5-HT3 antagonist. Granulocyte colony-stimulating factor was permitted as a therapeutic intervention for neutropenia but not as prophylaxis. Subsequent doses were modi- fied based on hematological and non-hematological toxicities. If grade 4 neutropenia persisted for 4 days, febrile neutropenia, grade 4 thrombocytopenia or grade 3 or worse non-hematological toxicities arose, the amrubicin dosage was reduced at the discretion of the physician in charge.

\section{Evaluation of response and toxicity}

Tumor responses were classified according to the Response Evaluation Criteria for Solid tumors. The patients were evaluated to determine the stage of their disease before starting treatment and at the time of determination of disease progression or relapse, by complete medical history and physical examination, chest X-ray, computed tomography (CT) of the chest and abdomen, CT or magnetic resonance imaging (MRI) of the head, and positron-emission tomography (PET). Enrolled patients with SCLC were stratified by type of relapse: refractory relapse, defined as relapse during the first-line chemotherapy or within 90 days after the completion of first-line chemotherapy, and sensitive relapse, defined as relapse $\geq 90$ days after the completion of first-line chemotherapy. Toxicities were assessed according to the Common Terminology Criteria for Adverse Events (CTCAE) version 3.0.

\section{Statistical analysis}

Primary end-points of this study were toxicity analysis and response rate, and secondary end points were progression-free survival (PFS) and overall survival (OS). Baseline characteristics, response and toxicity rates between the two groups were compared by Fisher's exact test. Survival time was compared between the two groups using the log-rank test. The mean number of treatment cycles, mean dose of amrubicin and mean interval of amrubicin administration were compared between the two groups using the two-tailed Student's t-test. Overall survival was calculated from the date of the first cycle of amrubicin monotherapy to the date of death from any cause or the last follow up. Progression-free survival was calculated from the date of the first cycle of amrubicin monotherapy to the date of disease progression, recurrence, or death from any cause. Probability values of $<0.05$ were considered significant.

\section{Results}

\section{Patients characteristics and treatment}

We enrolled 51 patients (NSCLC $n=21$; SCLC, $n=30$ ) between July 2003 and October 2009. The clinical characteristics of the patients are listed in Table 1 . Among the 21 patients with NSCLC, $16(76.2 \%)$ were $<70$ years of age, (median, 63.5 years; range, 39-69 years), and 5 (23.8\%) were $\geq 70$ years (median, 71 years; range, $71-75$ years). Of the 30 patients with SCLC, $13(43.3 \%)$ were $<70$ years of age (median, was 64 years; range, 51-67 years) and 17 (56.7\%) were $\geq 70$ years, (median, 74 years; range, 70-80 years). The male to female ratio, PS, histology, clinical stage and 
Table 1. Patient Characteristics

\begin{tabular}{|c|c|c|}
\hline Characteristic & $\begin{array}{l}\text { Age }<70 \text { years } \\
(n=29)\end{array}$ & $\begin{array}{l}\text { Age } \geq 70 \text { years } \\
(n=22)\end{array}$ \\
\hline Median age (range) & $64(39-69)$ & $73(70-80)$ \\
\hline \multicolumn{3}{|l|}{ Sex } \\
\hline Male & 21 & 19 \\
\hline Female & 8 & 3 \\
\hline \multicolumn{3}{|l|}{ PS (ECOG) } \\
\hline 0 & 5 & 2 \\
\hline 1 & 19 & 14 \\
\hline 2 & 5 & 5 \\
\hline 3 & 0 & 1 \\
\hline \multicolumn{3}{|l|}{ Histology } \\
\hline adenocarcinoma & 10 & 2 \\
\hline squamouscell carcinoma & 5 & 2 \\
\hline NSCLC not spcified & 1 & 1 \\
\hline SCLC & 13 & 17 \\
\hline \multicolumn{3}{|l|}{$\begin{array}{l}\text { Stage } \\
\text { (NSCLC) }\end{array}$} \\
\hline IIIA & 1 & 0 \\
\hline IIIB & 4 & 1 \\
\hline $\begin{array}{l}\text { IV } \\
(\mathrm{SCLC})\end{array}$ & 11 & 4 \\
\hline LD & 2 & 2 \\
\hline ED & 11 & 15 \\
\hline \multicolumn{3}{|l|}{$\begin{array}{l}\text { Number of prior regimens } \\
\text { (NSCLC) }\end{array}$} \\
\hline$\leq 3$ & 8 & 3 \\
\hline $\begin{array}{l}\geq 4 \\
(\mathrm{SCLC})\end{array}$ & 8 & 2 \\
\hline 1 & 5 & 11 \\
\hline 2 & 7 & 6 \\
\hline 3 & 1 & 0 \\
\hline
\end{tabular}

NSCLC: Non-small Cell Lung Cancer, SCLC: Small Cell Lung Cancer, ECOG: Eastern Cooperative Oncology Group, PS: Performance Status, LD: Limited Disease, ED: Extensive Disease

number of prior regimens did not significantly differ between the two age groups. Of 30 patients with SCLC, type of relapse was as follows: $11(36.7 \%)$ were sensitive relapse, and 19 (63.3\%) were refractory relapse. The type of relapse did not significantly differ between the two groups.

\section{Toxicity}

Toxicity was evaluated in all treated patients. Overall, 134 courses were administered to 51 (29 younger and 22 elderly) patients. The overall mean number of treatment cycles was 2.63 , the mean dose of amrubicin was $36.00 \mathrm{mg} / \mathrm{m}^{2} /$ day, and the mean interval between amrubicin doses was 30.92 days. Mean numbers of treatment cycles (2.83 and 2.36 for age $<70$ and $\geq 70$ years, respectively; $\mathrm{p}=0.417$ ), mean doses of amrubicin ( 37.35 vs. $35.87 \mathrm{mg} / \mathrm{m}^{2} /$ day; p=0.062) and mean interval of administration (30.49 vs. 31.67 days; $p=$ 0.552) did not significantly differ between the two age groups (Table 2). The hematological toxicity is summarized in Table 3 and shows that the incidence of grade 3 or 4 hematological toxicity did not differ between the younger and older groups (leukopenia, $48.3 \%$ and $59.1 \%$ for age $<70$ and $\geq 70$ years, $\mathrm{p}=0.573$; neutropenia, $65.5 \%$ vs. $77.3 \%$, $\mathrm{p}=$ 0.536 ; anemia, $20.7 \%$ vs. $22.7 \%, \mathrm{p}=1.000$; thrombocytopenia, $13.8 \%$ vs. $31.8 \%, \mathrm{p}=0.173$; febrile neutropenia, $20.7 \%$ vs. $22.7 \%, \mathrm{p}=1.000)$. The non-hematological toxicity is summarized in Table 4 . The incidence of grade 3 or 4 non-hematological toxicity was very low, and mostly mild or moderate and reversible. One patient in the elderly group developed acute myocardial infarction. The rate of grade 2-4 non-hematological toxicity did not significantly differ between the two age groups.

\section{Response to therapy and survival}

The objective tumor responses of the two age groups are shown in Table 5. Among the 21 patients with NSCLC, the disease control rate (rates of patients who achieved complete or partial responses or stable disease), median PFS, and median survival time (MST) were 61.9\%, 49 and 304 days, respectively. Only one younger patient with NSCLC achieved a partial response. Of the 30 patients with SCLC, the response rate (rate of patients who achieved a complete or partial response), median PFS, and MST were 53.3\%, 86 days and 301 days, respectively. The response rates for patients with chemotherapy-sensitive and -refractory relapse SCLC were $54.5 \%$ and $47.4 \%$, respectively. Among 21 patients with NSCLC, the median values in the younger and elderly groups for PFS were 49.5 and 48.0 days, respectively, and for MST were 180 and 401 days, respectively. Among 30 patients with SCLC, the median values in the younger and elderly groups for PFS were 166 and 61 days, 
Table 2. Chemotherapy Delivery by Age Group

\begin{tabular}{|c|c|c|c|c|}
\hline & $\begin{array}{l}\text { All patients } \\
\quad(n=51)\end{array}$ & $\begin{array}{c}\text { Age }<70 \text { years } \\
(n=29)\end{array}$ & $\begin{array}{c}\text { Age } \geq 70 \text { years } \\
(n=22)\end{array}$ & $p$-value* \\
\hline mean chemotherapy cycles (range) & 2.63 & $2.83(1-10)$ & $2.36(1-9)$ & 0.417 \\
\hline mean amrubicin dose $\left(\mathrm{mg} / \mathrm{m}^{2} /\right.$ day) (range) & 36.00 & $37.35(30-45)$ & $35.87(30-40)$ & 0.062 \\
\hline mean interval of amrubicin administration (days) (range) & 30.92 & $30.49(21-71)$ & $31.67(21-73)$ & 0.765 \\
\hline total chemotherapy cycles & 134 & 82 & 52 & \\
\hline
\end{tabular}

*Student's T-test.

Table 3. Hematological Toxicities

\begin{tabular}{|c|c|c|c|c|c|c|c|c|c|c|c|c|}
\hline \multirow[t]{2}{*}{ Toxicity } & \multicolumn{6}{|c|}{ Age $<70$ years $(n=29)$} & \multicolumn{5}{|c|}{ Age $\geq 70$ years $(n=22)$} & \multirow[t]{2}{*}{$p$-value* } \\
\hline & 2 & 3 & 4 & No. & & $\%$ & 2 & 3 & 4 & No. & $\%$ & \\
\hline Leukopenia & 6 & 8 & 6 & & 14 & 48.3 & 7 & 4 & 9 & 13 & 59.1 & 0.573 \\
\hline Neutropenia & 7 & 5 & 14 & & 19 & 65.5 & 3 & 9 & 8 & 17 & 77.3 & 0.536 \\
\hline Anemia & 5 & 6 & 0 & & 6 & 20.7 & 5 & 5 & 0 & 5 & 22.7 & 1.000 \\
\hline Febrile neutropenia & - & 6 & 0 & & 6 & 20.7 & - & 5 & 0 & 5 & 22.7 & 1.000 \\
\hline
\end{tabular}

${ }^{*}$ Fisher's Exact Test (Grade $\leqq 2$ Vs. $\geqq 3$ ).

Table 4. Nonhematological Toxicities

\begin{tabular}{|c|c|c|c|c|c|c|c|c|c|c|c|c|}
\hline \multirow{2}{*}{ Toxicity } & \multicolumn{3}{|l|}{ Grade } & \multicolumn{2}{|l|}{$\geq$ Grade2 } & \multicolumn{3}{|l|}{ Grade } & \multicolumn{3}{|c|}{$\geq$ Grade 2} & \multirow{2}{*}{$p$-value* } \\
\hline & 2 & 3 & 4 & No. & $\%$ & 2 & 3 & 4 & No. & & $\%$ & \\
\hline Vomiting & 0 & 0 & 0 & 0 & 0 & 1 & 0 & 0 & & 1 & 4.5 & 0.431 \\
\hline Anorexia & 10 & 1 & 0 & 11 & 37.9 & 6 & 1 & 0 & & 7 & 31.8 & 0.770 \\
\hline Stomatitis & 0 & 0 & 0 & 0 & 0 & 1 & 0 & 0 & & 1 & 4.5 & 0.431 \\
\hline lleus & 0 & 1 & 0 & 1 & 3.4 & 0 & 0 & 0 & & 0 & 0 & 1.000 \\
\hline Epilation & 2 & 0 & 0 & 2 & 6.9 & 1 & 0 & 0 & & 1 & 4.5 & 1.000 \\
\hline Hyponatremia & 0 & 1 & 0 & 1 & 3.4 & 0 & 1 & 0 & & 1 & 4.5 & 1.000 \\
\hline AMI & 0 & 0 & 0 & 0 & 0 & 0 & 0 & 1 & & 1 & 4.5 & 0.430 \\
\hline
\end{tabular}

AMI: Acute Myocardial Infarction *Fisher's Exact Test (Grade $\leqq 1$ Vs. $\geqq 2$ ).

Table 5. Response by Age Group

\begin{tabular}{|c|c|c|c|c|c|c|}
\hline \multicolumn{4}{|c|}{ Response NSCLC } & \multicolumn{3}{|l|}{ SCLC } \\
\hline & $\begin{array}{l}\text { Age }<70 \text { years } \\
(n=16)\end{array}$ & $\begin{array}{l}\text { Age } \geq 70 \text { years } \\
(n=5)\end{array}$ & $p$-value* & $\begin{array}{l}\text { Age }<70 \text { years } \\
(n=13)\end{array}$ & $\begin{array}{l}\text { Age } \geq 70 \text { years } \\
(n=17)\end{array}$ & $p$-value* \\
\hline$\overline{C R}$ & 0 & 0 & - & 1 & 0 & - \\
\hline PR & 1 & 0 & - & 7 & 8 & - \\
\hline SD & 10 & 2 & - & 5 & 3 & - \\
\hline PD & 5 & 3 & - & 0 & 6 & - \\
\hline $\mathrm{CR}+\mathrm{PR}$ & $1(6.3 \%)$ & $0(0 \%)$ & 1.000 & $8(61.5 \%)$ & $8(47.1 \%)$ & 0.484 \\
\hline $\mathrm{CR}+\mathrm{PR}+\mathrm{SD}$ & $11(68.8 \%)$ & $2(40 \%)$ & 0.325 & $13(100 \%)$ & $11(64.7 \%)$ & 0.024 \\
\hline
\end{tabular}

NSCLC: Non-small Cell Lung Cancer, SCLC: Small Cell Lung Cancer, CR: Complete Response, PR: Partial Response, SD: Stable Disease, PD: Progressive Disease. *Fisher's Exact Test. 
respectively, and for MST were 340 and 279 days, respectively. The response rates of SCLC $(61.5 \%$ and $47.1 \%, \mathrm{p}=$ $0.484)$, and the disease control rates of NSCLC (68.8\% vs. $40 \%, \mathrm{p}=0.325$ ) did not significantly differ between the two groups. Neither PFS nor OS significantly differed between the younger and elderly patients with NSCLC (PFS, $\mathrm{p}=$ 0.563; OS, $\mathrm{p}=0.499)$. However, PFS and OS statistically differed in both of the age groups with SCLC (PFS, $\mathrm{p}=0.039$; OS, $\mathrm{p}=0.015$ ).

\section{Discussion}

This study retrospectively analyzed the effect and safety of amrubicin monotherapy in patients with recurrent or refractory lung cancer who received amrubicin after previous chemotherapy. Amrubicin toxicity was acceptable for elderly patients with previously treated lung cancer.

The number of elderly patients with lung cancer will likely increase with the rapidly growing geriatric population $(13,14)$. Elderly patients with good PS and normal organ functions tend to be treated with regimens similar to those of younger patients, but some reports suggest that elderly patients even with good PS and normal organ functions might be at greater risk of severe toxicity than younger patients $(11,15,16)$. Therefore, elderly patients with NSCLC were much less likely to receive chemotherapy, and more likely to receive non-platinum chemotherapy regimens (11). Similarly, non-cisplatin regimens such as carboplatin and etoposide combination become one of the standard chemotherapy regimens for elderly patients with SCLC (17). Furthermore, treatment options for elderly patients with recurrent or refractory lung cancer remain limited. Amrubicin is often administered to patients with refractory or recurrent lung cancer, but whether amrubicin monotherapy for elderly patients with previously treated lung cancer is clinically safe has not been demonstrated. This is the first analysis of the safety and benefit of amrubicin for elderly patients with previously treated lung cancer.

Some recent reports have indicated that amrubicin monotherapy is an active agent for the treatment of refractory or relapsed SCLC, with manageable toxicities $(6,7)$. In addition, some authors have described positive effects and acceptable toxicity of amrubicin monotherapy in previously treated patients with $\operatorname{NSCLC}(3,5)$. Amrubicin as a single agent was approved at a dosage of $45 \mathrm{mg} / \mathrm{m}^{2} /$ day in chemotherapy-naïve patients with lung cancer. However, a reduced dose is administered to previously treated patients due to hematological toxicity. Reported studies recommend an amrubicin dose of $35 \mathrm{mg} / \mathrm{m}^{2} /$ day in patients with previously treated lung cancer $(2,3,18)$. We administered younger and elderly patients with mean amrubicin doses of 37.35 and $35.87 \mathrm{mg} / \mathrm{m}^{2} /$ day, respectively, which we consider is acceptable.

The toxicity profile of amrubicin in the present study was reasonable, in accordance with a previous study that noted myelosuppression as the major toxic effect (1-7). Mean- while, non-hematological types of toxicity were generally mild, and tended to be similar. The frequency of grades 3 and 4 leukopenia, neutropenia, anemia, thrombocytopenia, febrile neutropenia and grades 2-4 non-hematological toxicity did not significantly differ between the two age groups. The incidence of fatal toxicity was rare, and almost all types of toxicity should be manageable in both age groups. These findings suggest that the toxicity profile of amrubicin 30-40 $\mathrm{mg} / \mathrm{m}^{2} /$ day is acceptable for elderly patients with previously treated lung cancer. Only one patient who had a history of cerebral infarction in the elderly group developed acute myocardial infarction on day 30 of the chemotherapy, and we decided to discontinue the amrubicin monotherapy of that patient. Although amrubicin is classified as anthracycline, myocardial toxicity has not been reported in previous clinical trials. It is unclear whether acute myocardial infarction noted in that patient was a result of amrubicin monotherapy, but careful consideration may be needed when amrubicin is given to elderly patients with a risk of cardiovascular event.

In the present study, the response rates of SCLC (53.3\%) and the disease control rate of NSCLC (61.9\%) for amrubicin monotherapy were similar to those reported by others (SCLC response rates, 38.0-53.1\%; NSCLC control rate $33.3-62.2 \%$ ) (1-7). Moreover, regarding the response rate for patients with chemotherapy-sensitive and -refractory relapse SCLC $(54.5 \%$ and $47.4 \%$, respectively), the result of our study was reasonable in accordance with previous studies $(2,6,7)$. The response rate of SCLC and disease control rate of NSCLC did not significantly differ between the two age groups, indicating that amrubicin monotherapy is equally effective in elderly and younger patients. However, only one patient with NSCLC achieved a partial response, so amrubicin monotherapy did not seem to confer an obvious benefit for patients with NSCLC. Furthermore, six elderly patients with SCLC showed a progressive disease (PD), but none of the younger patients with SCLC showed a PD. Neither PFS nor OS significantly differed between the two age groups with NSCLC, but both were shorter in the elderly than in the younger group of SCLC patients. Because this study of a small sample of patients was based on clinical data from a single facility, larger prospective trials of elderly patients with recurrent or refractory lung cancer treated with amrubicin monotherapy should be conducted to determine the true response and survival.

In conclusion, amrubicin monotherapy might be an active and tolerable regimen for elderly patients with previously treated lung cancer. The information presented herein might provide a new direction for clinical research on the treatment of elderly patients with lung cancer after one or more chemotherapy regimens.

\section{References}

1. Kurata T, Okamoto I, Tamura K, Fukuoka M. Amrubicin for nonsmall-cell lung cancer and small-cell lung cancer. Invest New Drugs 25: 499-504, 2007. 
2. Shimokawa T, Shibuya M, Kitamura K, et al. Retrospective analysis of efficacy and safety of amrubicin in refractory and relapsed small-cell lung cancer. Int J Clin Oncol 14: 63-69, 2009.

3. Igawa S, Takahashi T, Nakamura Y, et al. Efficacy of amrubicin for non-small cell lung cancer after failure of two or more prior chemotherapy regimens. Anticancer Res 28: 3855-3858, 2008.

4. Kaneda H, Okamoto I, Hayashi H, et al. Phase II trial of amrubicin for second-line treatment of advanced non-small cell lung cancer: results of the West Japan Thoracic Oncology Group trial (WJTOG0401). J Thorac Oncol 5: 105-109, 2010.

5. Kaira K, Sunaga N, Tomizawa Y, et al. A phase II study of amrubicin, a synthetic 9-aminoanthracycline, in patients with previously treated lung cancer. Lung Cancer 69: 99-104, 2010.

6. Onoda S, Masuda N, Seto T, et al. Phase II trial of amrubicin for treatment of refractory or relapsed small-cell lung cancer: Thoracic Oncology Research Group Study 0301. J Clin Oncol 24: 5448-5453, 2006.

7. Inoue A, Sugawara S, Yamazaki K, et al. Randomized phase II trial comparing amrubicin with topotecan in patients with previously treated small-cell lung cancer: North Japan Lung Cancer Study Group Trial 0402. J Clin Oncol 26: 5401-5406, 2008.

8. Bunn PA Jr, Lilenbaum R. Chemotherapy for elderly patients with advanced non-small-cell lung cancer. J Natl Cancer Inst 95: 341343, 2003.

9. Gridelli C, Shepherd FA. Chemotherapy for elderly patients with non-small cell lung cancer: a review of the evidence. Chest 128: 947-957, 2005.

10. Sekine I, Fukuda H, Kunitoh H, Saijo N. Cancer chemotherapy in the elderly. Jpn J Clin Oncol 28: 463-473, 1998.

11. Chrischilles EA, Pendergast JF, Kahn KL, et al. Adverse events among the elderly receiving chemotherapy for advanced nonsmall-cell lung cancer. J Clin Oncol 28: 620-627, 2010.

12. Jian L, Ping C, Chun-Hua D, Xiao-Qin L, Qian-Lei B. Outcome and treatment in elderly patients with small cell lung cancer: A retrospective study. Geriatr Gerontol Int 9: 172-182, 2009.

13. Sekine I, Yamamoto N, Kunitoh H, et al. Treatment of small cell lung cancer in the elderly based on a criteria literature review of clinical trials. Cancer Treat Rev 30: 359-368, 2004.

14. Stephens RJ, Johnson DH. Treatment and outcomes for elderly patients with small cell lung cancer. Drugs Aging 17: 229-247, 2000.

15. Jara C, Gómez-Aldaraví JL, Tirado R, Meseguer VA, Alonso C, Fernández A. Small-cell lung cancer in the elderly--is age of patient a relevant factor? Acta Oncol 38: 781-786, 1999.

16. Oshita F, Kurata T, Kasai T, et al. Prospective evaluation of the feasibility of cisplatin-based chemotherapy for elderly lung cancer patients with normal organ functions. Jpn J Cancer Res 86: 11981202, 1995.

17. Okamoto H, Watanabe $\mathrm{K}$, Kunikane $\mathrm{H}$, et al. Randomised phase III trial of carboplatin plus etoposide vs. split doses of cisplatin plus etoposide in elderly or poor-risk patients with extensive disease small-cell lung cancer: JCOG 9702. Br J Cancer 97: 162169, 2007.

18. Okamoto I, Hamada A, Matsunaga Y, et al. Phase I and pharmacokinetic study of amrubicin, a synthetic 9-aminoanthracycline, in patients with refractory or relapsed lung cancer. Cancer Chemother Pharmacol 57: 282-288, 2006.

(C) 2010 The Japanese Society of Internal Medicine http://www.naika.or.jp/imindex.html 